with complete cytoreduction. In this study, we evaluated the survival impact of cardiophrenic lymph node enlargement in women with advanced stage epithelial ovarian cancer who have undergone cytoreductive surgery.

Methodology The Embase, Medline, Web of science, Cochrane Library and Google scholar databases were searched for articles from the database inception to November 2020. Metaanalysis was conducted to determine the prognostic impact of surgical outcome, postoperative complication and survival using random-effects models.

Result(s)* Fifteen relevant studies, involving 727 patients with CPLN adenopathy and 981 patients without CPLN adenopathy, were included in the review. The prevalence of ascites, and intra and extra abdominal metastases were highest in the CPLN adenopathy group. The mean size of pre-operative CPLN was $9.1 \pm 3.75 \mathrm{~mm}$. Eighty-two percent of the patients with CPLN resection, the resected CPLN were histological confirmed pathologic nodes. Surgical outcomes and perioperative complications did not differ between both groups. The pooled median overall survival (OS) was 42.7 months (95\% CI 10.8-74.6) versus 47.3 months (95\% CI 23.2-71.2) in patients with and without CPLN adenopathy. The pooled median progression free survival (PFS) was 14.6 months $(95 \%$ CI 4.9-24.4) versus 27.8 months (95\% CI 3.2-52.5) respectively. Patients with CPLN adenopathy had a significantly increased risk of disease recurrence (OR4.56, 95\% CI 1.9810.51, $\mathrm{P}<0.001$ ) and of dying from disease (OR 2.96, 95\% CI 2.08- 4.22, $\mathrm{p}<0.001)$ compared to those without CPLN adenopathy.

Conclusion* Patients with CPLN adenopathy had a higher tumor burden in both intra and extra-abdominal sites, and lower survival compared to patients without CPLN adenopathy. The available data was not sufficient to definitively confirm a therapeutic role of CPLN resection. A randomized controlled trial is needed to demonstrate the benefit of CPLN resection in cytoreductive surgery.

\section{IMPACT OF DISEASE PROGRESSION ON HEALTH- RELATED QUALITY OF LIFE OF ADVANCED OVARIAN CANCER (AOC) PATIENTS - POOLED ANALYSIS FROM THE PRIMA TRIAL}

${ }^{1} \mathrm{DM}$ Chase ${ }^{*},{ }^{2} \mathrm{M}$ Romeo Marin, ${ }^{3} \mathrm{~F}$ Backes, ${ }^{4} \mathrm{~S}$ Han, ${ }^{5} \mathrm{~W}$ Graybill, ${ }^{6} \mathrm{~B}$ Lund, ${ }^{7} \mathrm{~B}$ Pothuri, ${ }^{8} \mathrm{G}$ Mangili, ${ }^{9} \mathrm{D}$ O'malley, ${ }^{10} \mathrm{D}$ Berton, ${ }^{11} \mathrm{~L}$ Willmott, ${ }^{12} \mathrm{~K}$ Baumann, ${ }^{13} \mathrm{R}$ Coleman, ${ }^{14} \mathrm{~T}$ Safra, ${ }^{15} \mathrm{~V}$ Heinzelmann-Schwarz, ${ }^{16} \mathrm{D}$ Lorusso, ${ }^{17} \mathrm{~F}$ Karl, ${ }^{18} \mathrm{~T}$ Woodward, ${ }^{1} \mathrm{BJ}$ Monk, ${ }^{19} \mathrm{~A}$ GonzalezMartin. 'GOG and Arizona Oncology (US Oncology Network), University of Arizona, Creighton University, Phoenix, AZ, USA; ${ }^{2}$ GEICO and Medical Oncology Department, Institut Català d'Oncologia, Badalona, Spain; ${ }^{3}$ The Ohio State University Wexner Medical Center, GOG and the Department of Obstetrics and Gynecology, Columbus, OH, USA; ${ }^{4}$ University Hospitals Leuven, BGOG and Department of Gynaecology and Obstetrics, Leuven, Belgium; ${ }^{5}$ Medical University of South Carolina, Gynecologic Oncology Group (GOG) and Department of Gynecologic Oncology, Charleston, SC, USA; ${ }^{6}$ NSGO and Aalborg University Hospital, Aalborg, Denmark; ' Laura and Isaac Perlmutter Cancer Center, NYU Langone Health, GOG and Department of Obstetrics/Gynecology, New York, NY, USA; ${ }^{8}$ MITO and IRCCS Ospedale San Raffaele, Milan, Italy; ${ }^{9}$ Ohio State University COM - James CCC, GOG and Division of Gynecologic Oncology, Columbus, OH, USA; ${ }^{10}$ GINECO and ICO, Nantes, France; ${ }^{11} \mathrm{GOG}-\mathrm{F}$ and Arizona Oncology Associates, Phoenix, AZ, USA; ${ }^{12} A G O$ and Klinikum der Stadt Ludwigshafen, Ludwigshafen Am Rhein, Germany; ${ }^{13}$ US Oncology Research (GOG), The Woodlands, TX, USA; ${ }^{14}$ ISGO and Tel Aviv Sourasky Medical Center and Sackler School of Medicine, Tel Aviv, Israel; ${ }^{15}$ SAKK and University Hospital Basel, Basel, Switzerland; ${ }^{16}$ MITO and Fondazione Policlinico Universitario Agostino Gemelli, IRCCS, Rome, Italy; ${ }^{17}$ GlaxoSmithKline, Berlin, Germany; ${ }^{18} \mathrm{GlaxoSmithKline,} \mathrm{Philadelphia,} \mathrm{PA,} \mathrm{USA;}{ }^{19}$ Clinica Universidad de Navarra, GEICO and Medical Oncology Department, Madrid, Spain
Introduction/Background* Clinical trials consistently demonstrate the detrimental impact of progressive disease (PD) on patients' health-related quality of life (HRQoL). Progressionfree survival (PFS) is an established regulatory endpoint. However, PFS is often excluded as an efficacy endpoint on the basis that - PFS is not patient relevant - in early benefit assessment by select EU health technology agencies. The PRIMA/ENGOT-OV26/GOG-3012 (NCT02655016) Phase 3 trial showed niraparib significantly prolongs median PFS vs placebo in patients with $\mathrm{AOC}$ responsive to $1 \mathrm{~L}$ platinum $(\mathrm{Pt}$ )based chemotherapy, (CT), regardless of biomarker status. This post-hoc analysis of PRIMA is the first study to examine the relationship between $\mathrm{HRQOL}$ and $\mathrm{PD}$ in a broad frontline AOC maintenance treatment setting.

Methodology In PRIMA, patients with AOC responsive to $1 \mathrm{~L}$ Pt CT were randomised 2:1 to once-daily, maintenance niraparib or placebo. Impact of PD on patient HRQoL, irrespective of treatment, was evaluated within the pooled ITT population by comparing HRQoL at the last on-treatment (pre-progression) visit with HRQoL at end of treatment (EOT), +4 weeks, +8w, +12w, and +24w. Assessments included FOSI, EORTC QLQ-C30, EQ-5D-VAS, and EORTC QLQ-OV28 abdominal/GI symptom scale. ANCOVA was applied with treatment as a fixed effect and HRQoL at last on-treatment visit as a continuous covariate. Mixed models for repeated measurements (MMRM) evaluated cumulative HRQoL changes.

Result(s)* Significant reductions in HRQoL from pre- to postprogression were observed across all measures. Compared with pre-progression, FOSI scores (Least-squares mean $[95 \%$ CI $]$ ) were lower at EOT $+4 \mathrm{w}(-2.2[-2.8,-1.6])$ and EOT $+24 \mathrm{w}(-$ $1.7[-2.3,-1.1])$; each $\mathrm{p}<0.0001$. Similarly, at these timepoints EORTC-QLQ-C30 scores were lower by $-10.2(-12.4,-8.0)$ and $-10.7(13.2,-8.2)$ points, respectively, and EQ-5D-VAS by $-8.2(-10.4,-6.0)$ and $-6.2(-8.2,-4.2)$ points, respectively; each $\mathrm{p}<0.0001$. EORTC QLQ-OV28 scores were significantly worse at EOT $+4 \mathrm{w}(6.6[4.3,8.9])$ and $\mathrm{EOT}+24 \mathrm{w}(5.0[2.8$, $7.2])$; each $p<0.0001$. Similar changes were seen on MMRM analysis.

Conclusion* These findings demonstrate HRQoL is negatively impacted by PD in AOC. Preservation of HRQoL, an important therapy goal in the maintenance setting particularly for asymptomatic patients, can be achieved with PFS prolongation. PFS is of significant relevance and clinically important for AOC patients.

\section{CLEAR CELL BORDERLINE OVARIAN TUMOR: CLINICAL CHARACTERISTICS, PROGNOSIS AND MANAGEMENT}

${ }^{1} \mathrm{G}$ Ricotta*, ${ }^{1} \mathrm{P}$ Morice, ${ }^{1} \mathrm{~A}$ Maulard, ${ }^{1} \mathrm{~S}$ Gouy, ${ }^{1} \mathrm{C}$ Genestie, ${ }^{2} \mathrm{M}$ Candiani, ${ }^{1} \mathrm{~A}$ Leary, ${ }^{1} \mathrm{C}$ Chargari, ${ }^{1} \mathrm{P}$ Pautier. ' Gustave Roussy, Villejuif, France; ${ }^{2}$ San Raffaele Hospital, Milano, Italy

\subsection{6/ijgc-2021-ESG0.495}

Introduction/Background* Most frequent borderline ovarian tumors are serous and mucinous subtypes. Clear Cell Borderline Ovarian Tumor (CCBOT) is one of the rarest subtypes of borderline ovarian malignancies. Very few are known about this subtype and most of reports concerns very short series or case reports. The aim of this study was to determine the prognosis of a series of CCBOT and to analyze data published in the literature about this rare disease. 